\section{Chronobiology and Nutrition}

\author{
Self-Reported Sleep Duration of Brazilian Adults and its Association with Circadian Dietary \\ Intakes-A Cross-Sectional Population-Based Study (P04-001)
}

Michelle A Castro, Marcela Garcez, and Regina M Fisberg

School of Public Health, University of São Paulo, Brazil

Objective: The aim of this study was to assess the association of self-reported sleep duration with circadian dietary intakes among Brazilian adults.

Methods: This cross-sectional study analyzed data from the 2015 Health Survey of São Paulo, Brazil, a population-based survey of 1081 free-living adults (aged $\geq 20 \mathrm{y}$ ). Individuals were asked about their sleep duration the previous night through the question: "How many hours and/or minutes of sleep did you get last night?" Sleep duration in hours was categorized as short $(\leq 6 \mathrm{~h})$, average $(7-8 \mathrm{~h})$, or long $(\geq 9 \mathrm{~h})$. Each individual's circadian dietary intake was assessed by one 24-h dietary recall according to the USDA Multiple-Pass Method, which involves reporting the time and name of the meals and describing the types and amounts of foods and beverages consumed in the preceding $24 \mathrm{~h}$. Nutrition Data System for Research software was used to obtain nutritional information. Multiple logistic and linear regression models were fitted to predict the adjusted probability of consuming meals and the average contribution of meals to total energy and nutrient intake according to categories of sleep duration. The significance level was set at $P<0.05$.

Results: Short- and long-duration sleepers represented 32\% and 19\% of adults, respectively. Short-duration sleepers began eating earlier (0758) and ended eating later in the day (2109) relative to long-duration ones. In addition, short-sleepers consumed more meals per day (4.2), had longer eating periods $(12.3 \mathrm{~h})$, and had longer intervals between meals $(3.1 \mathrm{~h})$. The adjusted probabilities of having breakfast (95\%) and snacks (81\%) were higher among shortduration sleepers. The average contributions of snacks to total energy (14\%), carbohydrates (8\%), protein $(2 \%)$, fat $(4 \%)$, and added sugar $(2 \%)$ were higher among short-duration sleepers. The contributions of dinner to energy (24\%), carbohydrates (12\%), protein (5\%), and fat (8\%) were higher among long-duration sleepers.

Conclusions: The circadian dietary intakes of Brazilian adults differed according to categories of sleep duration. Short sleep duration was associated with earlier and later daily meals, longer eating periods, higher probabilities of snack consumption, and larger contributions of these meals to total energy and macronutrient intakes, features that may contribute to diets having worse nutritional quality.

\section{Funding Sources}

Fundação de Amparo à Pesquisa do Estado de São Paulo (Fapesp) Conselho Nacional de Desenvolvimento Científico e Tecnológico (CNPq).

The Timing of Eating on Weekends Differs from Weekdays but Is Not Differently Associated with Weight Status (P04-002)

Lena L Ho, ${ }^{1}$ Susan Roberts, ${ }^{2}$ Carol J Boushey, ${ }^{3}$ Ashley Helvig, ${ }^{4}$ Owen H Maroney, ${ }^{5}$ Rashmi Sharma, ${ }^{5}$ and Megan A McCrory ${ }^{6}$

${ }^{1}$ Boston University, MA; ${ }^{2}$ Jean Mayer USDA Human Nutrition Research Center on Aging at Tufts University, MA; ${ }^{3}$ University of Hawaii Cancer Center, HI; and ${ }^{4}$ College of Nursing and Health Professions, Georgia State University, GA

Objectives: Meal timing has emerged as an important factor for obesity risk. However, few studies have addressed meal-timing differences on weekdays compared with weekends and their associations with adiposity. We hypothesized that people would eat later on weekends than

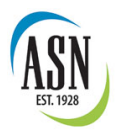

○

(c) 2018 American Society for Nutrition. All rights reserved.

Manuscript received September 20, 2017. Initial review completed March 23, 2018. Revision accepted May 27, 2018. Published online June 28, 2018 
weekdays, and that weekend meal timing would be more predictive of adiposity than that on weekdays.

Methods: Healthy, nonsmoking, weight-stable adults ( $n=125$; aged $18-64$ y, body mass index $18.9-36.2 \mathrm{~kg} / \mathrm{m}^{2}$ ) completed the Meal Pattern Grid modified to assess usual eating times, waking, and going-tosleep times during weekdays and weekends separately. Eating times were grouped into 8 time periods demarcating typical eating times: 0500-0859, 0900-1059, 1100-1259, 1300-1459, 1500-1659, 1700$1959,2000-2059$, and 2300-0459. We calculated the total number of daily eating occasions and durations of the overnight fast (last eating occasion before sleep to first eating occasion after waking), waking to the first eating occasion, and last eating occasion to sleep.

Results: On weekends, participants fasted longer overnight $(0.7 \pm 2.1 \mathrm{~h})$, ate more often during the night (2000-2059 and 2300-0459) ( $0.1 \pm 0.5$ and $0.2 \pm 0.7$ times), and less often in the early morning (0500-0859) (0.4 \pm 0.5 times) (mean \pm SD difference; all $P<0.01)$ than on weekdays. They also woke up later $(1.6 \pm 1.2 \mathrm{~h})$ and went to sleep later $(0.72 \pm 1.0 \mathrm{~h})(P<0.01)$ on weekends. Repeatedmeasures ANOVA, controlling for age, sex, and race/ethnicity, showed that weight status was positively related to the number of eating occasions per day and in the late afternoon (1300-1459), late night (2000-2059), and overnight (2300-0459) $(P=0.006$ to 0.05$)$ and inversely related to the number of hours fasting before sleeping $(P=0.032)$ and overnight $(P=0.09)$. Weight status was not related to weekday-weekend differences in wake or going-to-sleep times, and none of the above associations differed by weekday compared with weekend.

Conclusion: A shorter overnight fast, and eating closer to bedtime, more often in the late afternoon, late night, and overnight, were predictive of excess body weight regardless of weekday-weekend differences in meal timing.

Funding Sources

Supported by R01 DK075862 and Purdue University.

Associations among Timing of Food Intake, Eating Behavior Traits, and Psychobehavioral Factors in Overweight and Obese Individuals (P04-003)

Raphaelle Jacob, ${ }^{1}$ Angelo Tremblay, ${ }^{2}$ Véronique Provencher, ${ }^{2}$ Shirin Panahi, ${ }^{2}$ and Vicky Drapeau ${ }^{2}$

${ }^{1}$ Laval University, School of Nutrition, Institute of Nutrition and Functional Foods (INAF), Quebec Heart and Lung Institute Research Center, Quebec, Canada; and ${ }^{2}$ Laval University, Quebec, Canada

Background: Late distribution of food intake has recently been recognized as a determinant of obesity, and a few studies have observed that late eating is associated with a higher energy intake (EI). However, the mechanisms by which timing of food intake impacts body weight are not well understood.

Objective: The aim of this study was to assess the associations between distribution of food intake, eating, and psychobehavioral traits.

Methods: Overweight and obese individuals $(n=304$; age $38.7 \pm 8.4$ y; body mass index $33.2 \pm 3.4 \mathrm{~kg} / \mathrm{m}^{2} ; 55.3 \%$ women) who had participated in 4 previous studies were included in this cross-sectional study. EI was assessed with the use of a 3-d food record. The distribution of EI was assessed by calculating the percentage of total EI from period 5 (1700-1959) and period 6 (2000 until bedtime). Eating behavior traits were assessed with the Three-Factor Eating Questionnaire and the Binge Eating Scale, whereas psychobehavioral factors were evaluated through the use of the Pittsburgh Sleep Quality Index, the Body Esteem Scale, the Beck Depression Inventory, the State-Trait Anxiety Inventory, and the Perceived Stress Scale. Pearson correlations were used to assess the associations between distribution of EI and those variables among men and women.

Results: Among women, the \%EI from combined values for periods 5 and 6 was positively associated with disinhibition $(r=0.26$, $P=0.001)$ and with habitual and situational susceptibility to disinhibition ( $r=0.22, P=0.005$ and $r=0.18, P=0.03$, respectively). The \%EI from period 6 was positively associated with strategic dieting behavior $(r=0.17, P=0.03)$ in women. In men, the \%EI from periods 5 and 6 was negatively associated with susceptibility to hunger $(r=-0.19$, $P=0.03$ ), whereas the $\%$ EI from period 6 was positively correlated with susceptibility to hunger $(r=0.18, P=0.0496)$. The $\%$ EI from period 6 was also positively associated with depressive symptoms $(r=0.22$, $P=0.02)$, perceived stress $(r=0.23, P=0.01)$, and with state and traitanxiety $(r=0.22, P=0.02$ and $r=0.32, P=0.001$, respectively) in men.

Conclusions: The results of this study suggest that a higher proportion of EI consumed later in the day is associated with suboptimal eating and psychobehavioral traits and could contribute to explain the association between a delayed distribution of food intake and obesity.

\section{Funding Sources}

This study was partly funded by a grant of the Ministère de l'Enseignement supérieur, Recherche, Science et Technologie du Québec. AT is the holder of the Canada Research Chair in Environment and Energy Balance. $\mathrm{RJ}$ is a recipient of a $\mathrm{PhD}$ scholarship from the Fonds de Recherche du Québec-Santé. SP is the recipient of a postdoctoral fellowship from MITACS Accelerated.

Food Consumption of Rotating Shift Workers Is Influenced by the Interaction between Shift Day and Eating Duration (P04-004)

\section{Luisa P Marot Furlan, Dayane Rosa, Tassia VC Lopes, and Cibele Crispim}

\section{Federal University of Uberlandia, Brazil}

Background and Objective: Studies have reported that shift work rotation schedules may lead to irregular meal times among shift workers. However, the impact of the intense weekly variation of their routine on the food consumption pattern is unknown. The aim of this study was to evaluate if the interaction between shift days and eating duration (ED) - the interval between the first and the last meal of the day-influences energy and macronutrient intake during $10 \mathrm{~d}$ of a complete shift rotation schedule.

Methods: Thirty male shift workers were evaluated over $10 \mathrm{~d}$ in a shift schedule carried out as follows: $2 \mathrm{~d}$ during the day (D1 and D2), $2 \mathrm{~d}$ during the afternoon (D3 and D4), $24 \mathrm{~h}$ free day (D5), $2 \mathrm{~d}$ during the night (D6 and D7), and $72 \mathrm{~h}$ off (D8, D9, and D10). Dietary intake was evaluated by $24-\mathrm{h}$ recall (R24h) over all shifts of the schedule. The ED pattern was classified according to $\mathrm{R} 24 \mathrm{~h}$ as $<12 \mathrm{~h}$ or $\geq 12 \mathrm{~h}$. The effect of the interaction between ED pattern and shift day on energy and macronutrient intake was analyzed with the use of generalized estimating equations. 
Results: The interaction between ED pattern and shift days had an effect on energy and macronutrient intake $(P<0.05)$. Overall, the transition between the 24-h free day (D5) and the first night shift (D6) promoted an increase in energy (3160 kcal), fat (112.7 g), carbohydrate $(383.1 \mathrm{~g})$, and protein $(150.5 \mathrm{~g})$ intake, whereas the second night shift (D7) promoted a decrease in energy and macronutrient intake (1603 kcal, $P<0.001 ; 50.6$ g, $P<0.001 ; 220.5$ g, $P=0.026 ; 69.3$ g, $P<0.001$, respectively) for the $\mathrm{ED} \geq 12 \mathrm{~h}$ pattern. The same effect did not occur for $\mathrm{ED}<12 \mathrm{~h}$.

Conclusion: The interaction between shift day and ED had a significant contribution, with a poorer pattern of energy, carbohydrate, and fat consumption being observed with the $E D \geq 12 \mathrm{~h}$ pattern, mainly on the days that ended with night shifts. Thus, understanding the eating patterns of shift-workers is essential to develop nutritional strategies that will improve the eating habits and health status of this population.

Funding Sources

CAPES and FAPEMIG.

\section{Validity and Reliability of Two Food Timing Tools (P04-005)}

Heather Rasmussen, ${ }^{1}$ Prachi Chakradeo, ${ }^{2}$ Helen Burgess, ${ }^{2}$ Ali Keshavarzian, ${ }^{2}$ Lou Fogg, ${ }^{2}$ Garth Swanson, ${ }^{2}$ and Barbara Swanson $^{2}$

${ }^{1}$ University of Nebraska-Lincoln, NE; ${ }^{2}$ Rush University Medical Center, IL

Objectives: Circadian rhythms coordinate multiple processes over a 24-h day, including body temperature, metabolism, and immunity. Although the light-dark cycle is the main entrainer of the central circadian clock, food timing is an important entrainer of peripheral circadian rhythms, including the gastrointestinal tract (GIT). Thus, irregular eating could lead to GIT circadian misalignment. Easily administered, valid, and reliable tools to assess time of eating, and thus diet-induced circadian misalignment, do not currently exist. Therefore, the objective of this study was to test the validity and reliability of 2 tools that measure food and sleep timing: the food timing questionnaire (FTQ) and the food timing screener (FTS).

Methods: The content validity of the FTQ and FTS was assessed by an expert panel of 10 registered dietitian nutritionists. Each expert evaluated the clarity and relevance of the tools' items on a 4-point scale, and both an item content validity index (I-CVI) and scale content validity index (S-CVI) were calculated (range $0-1$ ). Internal consistency and stability were tested in a sample of 61 participants by calculating intraclass correlation coefficients (ICCs) and 48-h test-retest reliability. Criterion-related validity was tested by calculating Pearson's correlation between the FTQ and FTS with 2 validated instruments: ASA-24 recalls for food timing and the Munich Chronotype Questionnaire (MCTQ) for sleep timing.

Results: The sample was $72 \%$ female with a mean \pm SD age of $34.9 \pm 11.2 \mathrm{y}$ and mean \pm SD body mass index of $26.8 \pm 5.4 \mathrm{~kg} / \mathrm{m}^{2}$. All I-CVI scores ranged from 0.70 to 1.00 . The S-CVI values for the FTQ and FTS were 0.90 and 0.94 , respectively. All ICCs for the FTQ were $>0.51$ except for snacks, which ranged from 0.27 to 0.42 . All Pearson's correlations for the test-retest analysis of the FTQ and FTS were $>0.6$ except for snacks, which ranged from 0.09 to 0.49 . Pearson's correlations between MCTQ and FTQ were $>0.60$. There was a high correlation between the FTQ and the ASA-24 recalls except for lunch, which was 0.31 . Correlations between FTQ and FTS on all timings were $>0.6$ except for the first snack of the day.

Conclusions: Both instruments demonstrated validity and reliability, supporting their use in future studies examining the effects of food timing on health outcomes.

\section{Funding Sources}

None.

Relation between Timing of Food Intake and Intestinal Permeability and Inflammation (P04-006)

Heather Rasmussen, ${ }^{1}$ Ali Keshavarzian, ${ }^{2}$ Erika Davis, ${ }^{2}$ Robert

Hutkins, ${ }^{1}$ and Jens Walter ${ }^{3}$

${ }^{1}$ University of Nebraska-Lincoln, NE; ${ }^{2}$ Rush University Medical Center, IL; and ${ }^{3}$ University of Alberta, Canada

Objectives: An underappreciated disruptor of circadian rhythms is altered meal times, such as eating a majority of calories late in the day and inconsistent timing of food intake between days. As disrupted circadian rhythms may also adversely affect the gastrointestinal milieu, the objective of the current study was to explore the relation between food timing and markers of intestinal health in humans.

Methods: Participants who completed a food timing questionnaire (FTQ) at Rush University Medical Center were included in a crosssectional human study. Participants completed a 7-d FTQ, which included reporting time of both self-defined eating events (breakfast, lunch, dinner, and snacks) on both work and free days and selfdefined largest meal consumption. Inconsistent timing of food intake, or social jet lag, was defined as the difference in the average time of the largest meal of work and free days of $>2 \mathrm{~h}$. Intestinal permeability was measured by collection of 24-h urine after consumption of a sugar mixture and presented as sucralose excretion as a percentage of the oral dose. Differences in continuous variables were analyzed with KruskalWallis and Mann-Whitney tests, as appropriate.

Results: Participants $(n=111)$ had a mean \pm SD age of $43.5 \pm$ $11.4 \mathrm{y}$, were primarily female (73.0\%), and identified as black/African American (64.9\%). The majority of subjects reported dinner as the largest eating event of the day (59.5\%), followed by lunch $(23.4 \%)$. Those who reported dinner as their largest meal had higher intestinal permeability than those who reported lunch as their largest meal (24-h sucralose, $0.45 \%$ compared with $0.74 \%$, respectively; $P=0.013$ ). Among subjects who had defined work and free days $(64.8 \%, n=70)$, those who had social jet lag (22\%) were younger and had higher interleukin-6 compared to those who did not have social jet lag (2.2 compared with 1.8 $\mathrm{pg} / \mathrm{mL}$, respectively; $P=0.046$ ). No differences in lipopolysaccharide binding protein $(11,815$ compared with $10,920 \mathrm{ng} / \mathrm{mL}, P=0.07)$ or lipopolysaccharide (0.02 compared with $0.01 \mathrm{EU} / \mathrm{mL}, P=0.519)$ were seen by social jet lag status.

Conclusions: Consuming food at the wrong time may influence the relation between food intake and gastrointestinal health and systemic inflammation. This relation should be further investigated in a larger sample of humans to obtain a more comprehensive understanding of dietary intake.

\section{Funding Sources}

None. 